\section{Physical Activity}

\section{OP13 DIET AND PHYSICAL ACTIVITY LEVELS AMONG UK YOUTH}

doi:10.1136/jech-2012-201753.013

${ }^{1} \mathrm{~K}$ McAloney, ${ }^{1} \mathrm{H}$ Graham, ${ }^{2} \mathrm{~J}$ Hall, ${ }^{3} \mathrm{C}$ Law, ${ }^{4} \mathrm{~L}$ Platt, ${ }^{2} \mathrm{H}$ Wardle. ${ }^{1}$ Department of Health Sciences, University of York, York, UK; ${ }^{2}$ National Centre for Social Research, London, UK; ${ }^{3}$ Institute of Child Health, UCL, London, UK; ${ }^{4}$ Centre for Longitudinal Studies, Institute of Education, University of London, London, UK

Background Both physical activity levels and dietary behaviours have consistently been linked with the development of chronic disease, obesity and ill-health in both adult and youth samples. Nationally there are established recommendations of minimum levels of physical activity and fruit and vegetable consumption necessary to promote good health. Developing healthy behaviour is particularly important for young people, as adult behaviours that are detrimental or positive for health and well-being are often established in childhood and adolescence. This paper aims to investigate the prevalence of physical activity and fruit and vegetable consumption and to explore the co-occurrence of these behaviours among young people in the UK, using the newly available Understanding Society study.

Methods This study is part of an ongoing secondary analysis of Understanding Society: The UK Household Longitudinal Study. The analysis sample consisted of 4,395 young people aged 10 - 15 living in the UK in 2009/2010 who participated in the first wave of the study. The prevalence of physical activity and fruit and vegetable consumption within the sample, and patterns of co-occurrence across the two behaviours, were explored descriptively. Multinomial regression models were estimated to investigate the social patterning of the health behaviour co-occurrence patterns, using socio-demographic characteristics of the young person and mother. Results $85.2 \%$ of young people did not meet the government recommendation for fruit and vegetable consumption, reporting less than 5 portions of fruit and vegetable daily. $70.6 \%$ of young people did not meet the recommendation for participation in daily physical activity. A small minority did not consume any fruits and vegetables (5.0\%); and 6.4\% reported participating in physical activity less often than weekly. On examining the patterns across combinations of the two behaviours, most young people did not meet both recommendations (62\%), while only $6.1 \%$ of young people met both recommendations. Multinomial regression models indicated that gender, ethnicity and religion and socio-economic status were significantly associated with health behaviour patterns.

Conclusion This paper presents an analysis of the most recently available health-risk behaviour data on children from all four countries of the UK. The results indicate that a high proportion of young people do not achieve levels of fruit and vegetable consumption or levels of participation in physical activity considered to be a minimum for good health, and these two behaviours tend to co-occur. The implications of these behaviour patterns and the associations with socio-demographic characteristics are discussed.

\section{OP14 CHILDREN'S AND ADOLESCENTS' SEDENTARY BEHAVIOUR IN RELATION TO HOUSEHOLD SOCIOECONOMIC STATUS, INCOME, AND AREA DEPRIVATION : THE 2008 HEALTH SURVEY FOR ENGLAND}

doi:10.1136/jech-2012-201753.014

${ }^{1,2} \mathrm{~N}$ Coombs, ${ }^{3} \mathrm{~A}$ Rowland, ${ }^{1,2}$ E Stamatakis. ${ }^{1}$ UCL, Department of Epidemiology and Public Health, London, UK: ${ }^{2} P A R G$ (UCL Population Health Domain Physical Activity Research Group), London, UK; ${ }^{3}$ School of Health Sciences, University of South Australia, Australia

Background Sedentary behaviour (sitting) is an emerging cardiometabolic risk factor in young people. Little is known about how household socioeconomic position (SEP) and sedentary behaviour are associated in children and adolescents. The aim of this study was to assess the associations between SEP (including area-level deprivation) and sedentary behaviour in school-age children and adolescents.

Methods The study sample was 4034 participants aged 5-15 yrs participated in the 2008 Health Survey for England which collected information on SEP (household income, Registrar General's social class of the household reference person) and neighbourhood deprivation. Sedentary behaviour assessment (proxy parental measures for 5-12yrs; self-reported for 13-15yrs) included television viewing and other sitting during non-school times. Total sitting time was measured in a sub-sample ( $\mathrm{N}=611)$ using accelerometers. We examined the multivariable associations between each SEP indicator and each sedentary time indicator using generalised linear models. Whenever appropriate, models were adjusted for age, sex, body mass index (BMI), physical activity, accelerometer wear time and mutually adjusted for the other SEP indicators.

Results The mean age of the sample was 10 yrs ( \pm 3$), 2922$ participants were aged 5-12 and 1112 were aged 13-15yrs. Household income and social class were inversely associated with daily television times (i.e. the higher the SEP the lower the television viewing times), e.g. compared to participants from households in the bottom income quartile, those in the top quartile had 14 minutes /day less ( $95 \% \mathrm{CI}: 3$ to $25, \mathrm{p}=0.009)$ of television viewing. Non-TV sitting during non-school time was higher in nonmanual than in manual social class households by 14 minutes/day (7 to 20, $\mathrm{p}<0.001$ ). Total (accelerometry-assessed) sitting time was higher among participants from households in the top income half ( $\geq £ 23135 /$ year) by 22 minutes/day ( 7 to $37, p<0.00$ ) compared to those in the bottom half. Area deprivation was not associated with sedentary behaviour.

Conclusion Low socioeconomic position is linked with higher television times but with lower total (accelerometry-assessed) sitting, and non-TV sitting during non-school time in children and adolescents. Inferences from studies looking at socioeconomic position and specific indices of sedentary behaviour (e.g., TV time) in children and adolescents may not be generalizable to total sitting time.

\section{OP15 NEIGHBOURHOOD DEPRIVATION, LAND USE AND PHYSICAL ACTIVITY: COMBINING ACCELEROMETRY AND GLOBAL POSITIONING SYSTEMS}

doi:10.1136/jech-2012-201753.015

${ }^{1} \mathrm{M}$ Hillsdon, ${ }^{2,3} \mathrm{E}$ Coombes, ${ }^{2,3} \mathrm{~A}$ Jones, ${ }^{1} \mathrm{P}$ Griew, ${ }^{4} \mathrm{P}$ Wilkinson, ${ }^{4} \mathrm{~S}$ Hajat. ${ }^{1}$ Sport and Health Science, University of Exeter, Exeter, UK; ${ }^{2}$ School of Environmental Sciences, University of East Anglia, Norwich, UK; ${ }^{3}$ Centre for Diet and Activity Research, Institute of Public Health, Cambridge, UK; ${ }^{4}$ Public and Environmental Health Research Unit, London School of Hygiene \& Tropical Medicine, London, UK

Background Neighbourhood deprivation is associated with physical activity but it is not known whether the locations where physical activity takes place also varies by deprivation. The Forty Area Study (FAST) combined Global Positioning Systems (GPS) technology with accelerometery in UK adults to measure actual locations in which physical activity takes place. We evaluate what proportion of moderate to vigorous intensity physical activity (MVPA) is spent in different locations according to neighbourhood deprivation.

Methods 1084 adults from Northwest England took part. Each participant's activity levels were recorded for 7 days using an accelerometer. A quarter of our participants also wore a GPS device. The accelerometer and GPS data were integrated into a Geographical Information System (GIS) containing information on the participants' home locations and the locations where physical activity was recorded. We then examined the land uses where participants undertook their MVPA. 
Results Respondents in the most deprived neighbourhoods undertook a greater proportion of their MVPA in and around their home location, in buildings, on the road network and in shopping areas compared to respondents from the most affluent neighbourhoods. The proportion of MVPA undertaken in green space was considerably higher in the most affluent compared to the most deprived neighbourhoods (28.9\% vs. $8.8 \%$ ). The places where people undertake physical activity vary according to neighbourhood deprivation suggesting differences in the purpose of activity and access to places for physical activity.

Conclusion Understanding where people undertake their physical activity provides valuable insights into the types of land uses that are important for physical activity and how they vary according to an area based indicator of socio-economic status.

\section{OP16 HOW IS OBJECTIVELY MEASURED PHYSICAL ACTIVITY ASSOCIATED WITH FALLS AND FEAR OF FALLING IN OLDER COMMUNITY DWELLING MEN?}

doi:10.1136/jech-2012-201753.016

'BJ Jefferis, 'S Iliffe, ${ }^{3} \mathrm{~N}$ Kerse, ${ }^{2} \mathrm{D}$ Kendrick, ${ }^{4} \mathrm{~S}$ Trost, 'LT Lennon, 'S Ash, 'SG Wannamethee, 'R Morris, ${ }^{5} \mathrm{PH}$ Whincup. 'Primary Care \& Population Health, UCL, London, UK; ${ }^{2}$ Division of Primary Care, University of Nottingham, Nottingham, UK; ${ }^{3}$ Department of General Practice and Primary Health Care, University of Auckland, Auckland, New Zealand; ${ }^{4}$ Department of Nutrition and Exercise Sciences, Oregon State University, Corvallis, OR, USA; ${ }^{5}$ Division of Population Health Sciences and Education, St George's University of London, London, UK

Background Falls are a major cause of disability in older age and fear of falling (FOF) may limit physical activity. Associations between falls, fear of falling and objectively measured physical activity (PA) has been little studied, particularly in communitydwelling older adults.

Methods The study sample was men participating in a prospective, population-based cohort study in 24 British towns provided questionnaire information on a history of falls in the previous year, fear of falling, and other medical history and wore an Actigraph GT3X accelerometer for 7 days. Uniaxial accelerometer data were analysed in 60 s epochs, excluding bouts of $>60$ minutes of complete inactivity. Days with $>=600$ minutes valid wear time were included. PA intensity was categorised as sedentary: $<100$ counts/minute, light: 100-1952 counts/minute and moderate to vigorous PA (MVPA):>1952 counts/minute. Associations between activity levels and falls, or fear of falling, were estimated using hierarchical linear regression models accounting for clustering and controlling for confounders

Results A total of 1543 men with a mean age of 77(range 71-91y) wore the Actigraph; 1100 (72\%) had $>600$ minutes wear time on 5-7 days and questionnaire data. $21 \%(n=229)$ had fallen, $30 \%(n=68)$ received medical attention. Men who fell took fewer steps/day than men who did not: mean difference (adjusted for age, day order, month, wear time and town) $-504(95 \% \mathrm{CI}-216,-792)$ and spent more minutes sedentary $13(95 \% \mathrm{CI} 4,22)$, and less in light $-10(95 \% \mathrm{CI}-1,-19)$ and MVPA $-3.5(95 \% \mathrm{CI}-1.3,-5.8)$. In relative terms, the reduction in MVPA was greatest: $22 \%(95 \%$ CI 7,37$)$. Differences were explained by exercise self-efficacy and mobility limitations, but not by exercise outcome expectation. 13\% ( $n=141)$ men reported FOF, of whom $53 \%(n=74)$ had fallen in the past year. Men with FOF took markedly fewer steps than men without:$1325(95 \%$ CI $-1646,-1005)$, spent more minutes in sedentary $29(95 \% 18,40)$, and less in light activity $-21(95 \%$ CI $-32,-11)$ or MVPA $-9(95 \%$ CI $-11,-7)$. In relative terms, the reduction in MVPA was greatest:-72\%(95\%CI -93,-52). Differences were partly explained by exercise self-efficacy, mobility limitations, and to a lesser extent, exercise outcome expectation. There was no evidence that FOF had a greater impact on PA levels among men who had fallen compared to those who had not fallen.
Conclusion PA levels and in particular step counts and MVPA levels, were lower among men who had fallen in the past year, and even more markedly, among those who reported FOF.

\section{Mental Health I}

\section{OP17 DO LOW LEVELS OF PSYCHOLOGICAL DISTRESS PREDICT MORTALITY? EVIDENCE FROM AN INDIVIDUAL PARTICIPANT META-ANALYSIS OF TEN PROSPECTIVE COHORT STUDIES}

doi:10.1136/jech-2012-201753.017

${ }^{1-3} \mathrm{TC}$ Russ, ${ }^{4} \mathrm{E}$ Stamatakis, ${ }^{4} \mathrm{M}$ Hamer, ${ }^{1-3,5} \mathrm{JM}$ Starr, ${ }^{4} \mathrm{M}$ Kivimaki, ${ }^{3,4} \mathrm{GD}$ Batty. ${ }^{1}$ Scottish Dementia Clinical Research Network, NHS UK, Perth, UK; ${ }^{2}$ Alzheimer UK Dementia Research Centre, University of Edinburgh, Edinburgh, UK; ${ }^{3}$ Centre for Cognitive Ageing \& Cognitive Epidemiology, University of Edinburgh, Edinburgh, UK; ${ }^{4}$ Department of Epidemiology \& Public Health, UCL, London, UK; ${ }^{5}$ NHS Lothian, Edinburgh, UK

Background A series of studies have linked psychological distress (depression and anxiety) with cause-specific mortality, but the risk of lower, sub-clinically symptomatic, levels of distress - hitherto of little interest to specialist mental health practitioners - has not been quantified.

Methods We conducted an individual participant meta-analysis of ten large prospective cohort studies from the Health Survey for England. Baseline psychological distress was measured using the 12-item General Health Questionnaire, and mortality from death certification. The analytical sample comprised 68,222 individuals from general population samples of adults aged 35 and over, free of cardiovascular disease and cancer and living in private households in England at study baseline. The main outcome measures were death from all causes $(n=8,365)$, cardiovascular disease $(n=3,382)$, all cancers $(n=2,552)$, and external causes $(n=386)$. Mean (standard deviation) follow-up was 8.2 (3.5) years.

Results There was a positive, dose-response association between psychological distress across the full range of severity and mortality risk (age- and sex-adjusted hazard ratio for General Health Questionnaire scores of $1-3$ compared to zero: $1.20,95 \%$ CI 1.13 to 1.27 ; scores 4-6: 1.431 .31 to 1.56 ; and scores $7-12$ : $1.94,1.66$ to 2.26 ; $\mathrm{p}$ for trend $<0.001)$. This association remained after adjustment for somatic co-morbidity plus behavioural and socioeconomic factors. A similar association was found for death from cardiovascular disease, cancer, and external causes.

Conclusion Psychological distress is associated with increased risk of mortality from several major causes in a dose-response pattern. Even at lower levels of distress the mortality risk was elevated.

\section{OP18 CHILDLESSNESS IN EUROPE: IMPLICATIONS FOR WELLBEING IN LATER LIFE}

doi:10.1136/jech-2012-201753.018

1.2S Gibney. ${ }^{1}$ Centre for Behaviour and Health, Geary Institute, University College Dublin, Dublin, Ireland; ${ }^{2}$ School of Public Health, Physiotherapy and Population Science, University College Dublin, Dublin, Ireland

Background In addition to low patterns of fertility experienced in the last 15 years, lifetime childlessness is increasing in Europe. It is now expected that between 10-20\% of women born in the 1950s in Western Europe will never have children. Adult children play an important supportive role to their ageing parents; economically, acting as bridges to social support and monitoring health behaviour. This role has been enshrined at various institutional levels, from the family to the welfare state. Explanations of the negative relationship between childlessness and late life wellbeing have mainly focused on social support deficits. However, contradictory evidence of the effect of childlessness on psychological wellbeing exists which may be dependent on the wellbeing measures employed, the life course stage under 